\title{
Perceived Controllability Modulates the Neural Response to Pain
}

\author{
Tim V. Salomons, ${ }^{1,2}$ Tom Johnstone, ${ }^{1,3}$ Misha-Miroslav Backonja, ${ }^{4}$ and Richard J. Davidson ${ }^{1,2,3}$ \\ ${ }^{1}$ W. M. Keck Laboratory for Functional Brain Imaging and Behavior and Departments of ${ }^{2}$ Psychology, ${ }^{3}$ Psychiatry, and ${ }^{4}$ Neurology, University of \\ Wisconsin-Madison, Madison, Wisconsin 53706
}

\begin{abstract}
The response to painful stimulation depends not only on peripheral nociceptive input but also on the cognitive and affective context in which pain occurs. One contextual variable that affects the neural and behavioral response to nociceptive stimulation is the degree to which pain is perceived to be controllable. Previous studies indicate that perceived controllability affects pain tolerance, learning and motivation, and the ability to cope with intractable pain, suggesting that it has profound effects on neural pain processing. To date, however, no neuroimaging studies have assessed these effects. We manipulated the subjects' belief that they had control over a nociceptive stimulus, while the stimulus itself was held constant. Using functional magnetic resonance imaging, we found that pain that was perceived to be controllable resulted in attenuated activation in the three neural areas most consistently linked with pain processing: the anterior cingulate, insular, and secondary somatosensory cortices. This suggests that activation at these sites is modulated by cognitive variables, such as perceived controllability, and that pain imaging studies may therefore overestimate the degree to which these responses are stimulus driven and generalizable across cognitive contexts.
\end{abstract}

Key words: pain; control; imaging; insula; cingulate; cognitive

\section{Introduction}

It has long been acknowledged that the cognitive and affective context in which pain occurs plays a critical role in both the pain experience and the response that is elicited (Melzack and Casey, 1968). One contextual variable that is a critical determinant of the neural and behavioral response to nociceptive stimulation is the degree to which pain is perceived to be controllable. Perceived controllability, although not a physical property of the stimulus itself, affects pain tolerance (Maier and Watkins, 1998), learning and motivation (Mineka and Henderson, 1985), and the ability to cope with chronic pain (Jensen and Karoly, 1991; Jensen et al., 2001), suggesting that it has powerful effects on the way that nociceptive information is processed in the brain. To date, however, these effects have not been examined directly using neuroimaging techniques.

Previous pain imaging studies have identified a distributed neural network involved in pain processing, with the insular cortex, secondary somatosensory cortex (SII), and anterior cingulate cortex (ACC) being the most consistently activated areas (Peyron et al., 2000). These structures have been identified as part of an ascending interoceptive circuit that informs the organism about the current state of the body, indicating the location and intensity

Received April 7, 2004; revised May 27, 2004; accepted June 21, 2004.

This research was supported by Grant P50-MH069315 from the National Institute of Mental Health (R.J.D.). We thank Drew Fox for help with the preparation of figures, John Koger for assistance with programming, JongHoon "Jonnie" Lee for designing the field-mapping correction software applied to the data, and Keri Berling for general assistance. We thank Paul Whalen and Alex Shackman for comments on this manuscript.

Correspondence should be addressed to Richard J. Davidson, 1500 Highland Avenue, T225, Madison, WI 53705. E-mail: rjdavids@wisc.edu.

DOI:10.1523/JNEUROSCI.1315-04.2004

Copyright $\odot 2004$ Society for Neuroscience $\quad$ 0270-6474/04/247199-05\$15.00/0 of peripheral nociceptive input and generating a corresponding motivational state (Craig, 2003). With some notable exceptions (Rainville et al., 1997; Ploghaus et al., 1999; Bantick et al., 2002; Petrovic et al., 2002) (for review, see Petrovic and Ingvar, 2002; Rainville, 2002; Ploghaus et al., 2003), the extant neuroimaging literature has focused nearly exclusively on the patterns of activation produced in this network by peripheral stimulation. The goal of this study was to examine how the cognitive variable (perception of controllability) modifies these patterns of activation.

\section{Materials and Methods}

Subjects. Sixteen 19- to 35-year-old (mean, 22 years of age) right-handed subjects ( 11 males and 5 females) were recruited. Subjects were excluded if they were pregnant or claustrophobic, or had a present psychiatric or chronic pain disorder or significant history of such disorders. They were screened for medical conditions that could affect pain sensitivity and for regular use of drugs such as opioids or nonsteroidal anti-inflammatory drugs that could alter pain perception.

Study task. The experiment consisted of two sessions. In the first session, subjects chose a nociceptive stimulus (see below) and were given standardized instructions explaining the task. A scanner-compatible joystick was placed in the hand opposite to the side being stimulated. To familiarize subjects with the study task and the imaging environment, they were given the opportunity to rehearse the task in a mock scanner. In the second session, subjects were scanned while performing the same task.

We manipulated the perception of controllability by providing cues before the onset of painful thermal heat, which signaled whether the subject would be able to control the heat with a joystick response. Subjects were instructed that in the controllable condition (C), they could reduce the hot stimulus to a nonpainful duration ( $2 \mathrm{sec}$ instead of $5 \mathrm{sec}$ ). To do so, they were told that they had to meet two criteria. First, they had 

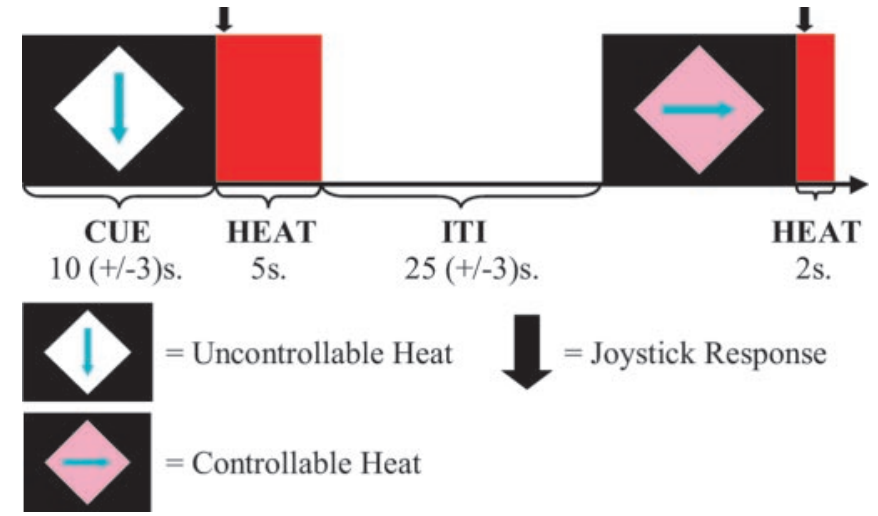

Figure 1. Study task. Cues were presented in which the color of the diamond indicated whether a subject could control the length of the heat. In the controllable condition, subjects were instructed that if they moved the joystick in the direction of the arrow and their response was faster than a threshold (based on their previous response times), they would receive the short $(2 \mathrm{sec})$ rather than long $(5 \mathrm{sec})$ heat. Subjects were asked to respond on all trials, regardless of whether they had control. One controllable and one uncontrollable trial are shown at the top. Each subject received eight trials of each condition. Colors were counterbalanced between subjects, and the direction of the arrow varied randomly within condition.

to respond in the correct direction, which was indicated by an arrow in the cue (Fig. 1). Arrows were presented in quasi-random order within each condition, so that subjects had to respond twice in each of four directions in each condition. The second criterion was that their response time had to be less than a "response threshold," which was based on their response time on previous trials. They were encouraged to try to beat this response threshold on every trial. In the uncontrollable condition (UC), subjects were asked to respond as they had in the controllable condition but were instructed that their response would have no effect on the length of the heat.

Subjects received eight presentations of each condition, in quasirandom order (i.e., two presentations of each condition within each of four runs). To further control for order effects, the order in which the conditions were presented was randomized within each run. To control for effects caused by uncertainty, subjects were told that the proportion of short and long stimuli in the uncontrollable condition would be based in an ongoing manner on their performance in the controllable condition, so that the probability of getting a long, painful stimulus on a given trial was the same in both conditions. In reality, the subjects only had the illusion of control in the controllable condition, because the order and ratio of short to long stimuli were preset in both conditions. This ensured that all subjects would receive the same number of long, painful stimuli (four in each condition). To maintain the illusion of control, when subjects were supposed to receive a short stimulus on a control trial but responded unusually slowly or incorrectly, the short stimulus was traded for the next long stimulus. Conversely, if they were supposed to receive a long stimulus and they responded unusually quickly, the long stimulus was traded for the next short stimulus. To compensate for the fact that near the end of the trial there was no more flexibility to "trade off" stimuli, subjects were instructed that they might find it more difficult to succeed toward the end of the trial, because of a changing threshold that took their previous responses into account.

Cues were $10 \pm 3 \mathrm{sec}$ in duration. Cue offset was coincident with the onset of the thermal stimulus. Subjects were asked to respond at stimulus onset. The duration of the intertrial interval (ITI; the time from stimulus offset to cue onset) was pseudorandomized with a mean of $25 \pm 5 \mathrm{sec}$. Pseudorandomization of the cue and ITI duration was used to prevent subjects from predicting stimulus onset based on temporal cues and to differentiate between the conditions in analysis.

Ten seconds after the offset of thermal stimuli (during the intertrial interval), subjects were asked to rate their pain on an 11 point visual analog scale in which 0 was "no pain" and 10 was "the worst pain imaginable." After completion of the study task, subjects were interviewed to determine whether they understood the meaning of the various cues. To determine whether they believed they had control in the controllable condition, they were asked to respond to the statement "The color of the diamond accurately indicated whether I had control over the length of the heat" on a five-point scale, where 1 represented "strongly disagree" and 5 represented "strongly agree."

Nociceptive stimulus. A thermal stimulator (TSA-II; Medoc Advanced Medical Systems, Haifa, Israel) was used to generate the painful heat. A $30 \times 30 \mathrm{~mm}$ magnetic resonance imaging (MRI)-compatible Peltier device was attached to one of the subject's forearms. The side of stimulation was chosen at random and counterbalanced across subjects (eight subjects were stimulated on the left and eight were stimulated on the right).

Before performing the study task, a level of nociceptive stimulation was chosen on the basis of the subject's own pain ratings. Stimulation began at $32^{\circ} \mathrm{C}$ and increased by $0.7^{\circ} \mathrm{C} / \mathrm{sec}$. Subjects were asked to stop the stimulation by pressing a button when their pain reached an 8 on an 11 point numeric rating scale, with 0 representing no pain and 10 representing the worst pain imaginable. This was repeated 10 times, with a $30 \mathrm{sec}$ break between each presentation. The mean temperature from the final five trials was used as the painfully hot stimulus. The maximum temperature used in the experiment was not allowed to exceed $49^{\circ} \mathrm{C}$, and subjects were excluded if their "hot, painful" stimulus was $<46^{\circ} \mathrm{C}$.

Functional MRI image acquisition. Images were acquired on a General Electric (Fairfield, CT) Signa 3.0 tesla high-speed imaging device with a quadrature head coil. Functional images consisted of $30 \times 4 \mathrm{~mm}$ sagittal echo-planar imaging (EPI) slices covering the entire brain $[1 \mathrm{~mm}$ interslice gap; $64 \times 64$ in-plane resolution; $240 \mathrm{~mm}$ field of view (FOV); repetition time, $2000 \mathrm{msec}$; echo time (TE), $30 \mathrm{msec}$; flip $=90^{\circ} ; 240$ image volumes per run]. Four EPI images with identical acquisition parameters but with TE values of $30,31,33$, and $36 \mathrm{msec}$, respectively, were also acquired, to be used in calculating magnetic field maps for use in image distortion correction. Immediately preceding acquisition of functional images, a whole-brain high-resolution T1-weighted anatomical scan (three-dimensional T1-weighted inversion recovery fast gradient echo; $256 \times 256$ in-plane resolution; $240 \mathrm{~mm}$ FOV; $124 \times 1.1 \mathrm{~mm}$ axial slices) was acquired. Functional images were collected in four $8 \mathrm{~min}$ runs.

Analysis of functional MRI data. Analysis was performed using FEAT [functional MRI (fMRI) Expert Analysis Tool], version 5.00 (Beckmann et al., 2003), part of FSL [Oxford Centre for Functional Magnetic Resonance Imaging of the Brain (FMRIB, Oxford, UK) Software Library; www.fmrib.ox.ac.uk/fsl]. Data preprocessing consisted of slice time correction, motion correction using MCFLIRT [Motion Correction using FMRIB's Linear Image Registration Tool (FLIRT)] (Jenkinson et al., 2002), and image distortion correction (using in-house software). Data were smoothed with a $5 \mathrm{~mm}$ full width at half maximum Gaussian blur and high-pass filtered with a $100 \mathrm{sec}$ cutoff. A separate regressor was derived for each experimental condition and the rating screen by convolving a stimulus-based binary boxcar function with an ideal hemodynamic response. The time-series data for each voxel were then modeled as the linear sum of all regressors. Contrasts between conditions were calculated from the model parameter estimates. The resulting contrast maps were registered to standardized [Montreal Neurological Institute (MNI)] space with FLIRT before mixed effect modeling of group data (subject as a random factor and condition as a fixed factor) using FLAME (FMRIB's local analysis of mixed effects). $Z$ statistic images were thresholded at $p<0.01$, with a cluster-based correction for multiple comparisons using Gaussian random field theory (Worsley et al., 1992; Friston et al., 1994; Forman et al., 1995).

\section{Results}

\section{Behavioral}

All subjects reported being aware of the meaning of the cues signaling controllable and uncontrollable pain. Most subjects reported a strong belief that they had control over the controllable stimulus but not the uncontrollable stimulus. In response to the statement "the color of the diamond accurately indicated whether I had control over the length of the heat" (where 1 represented "strongly disagree" and 5 represented "strongly agree"), 


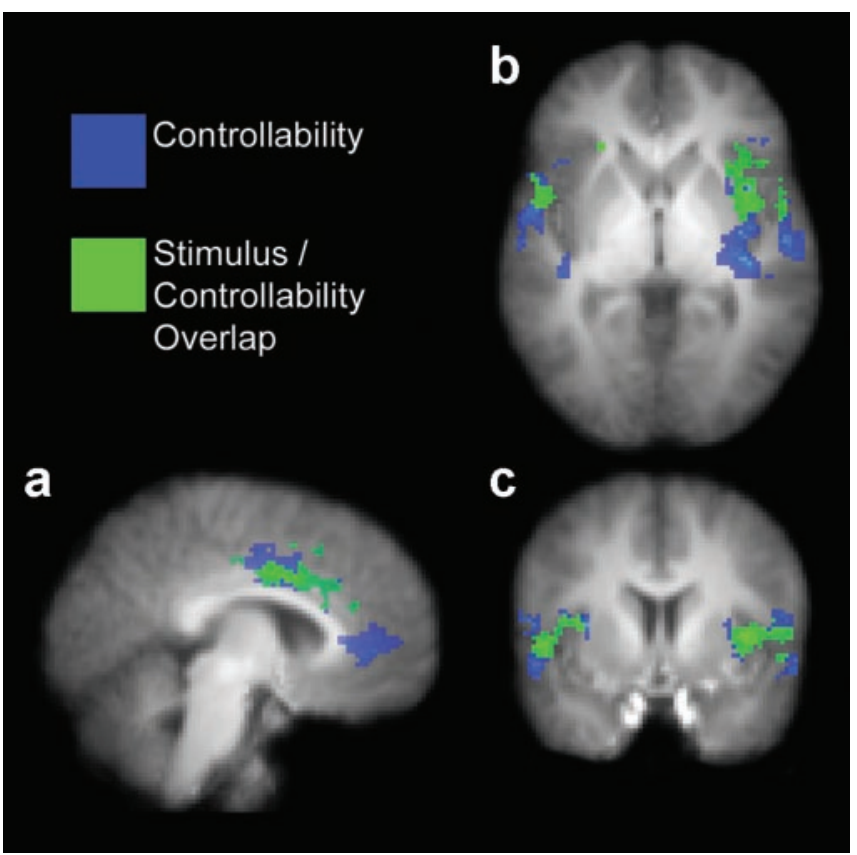

Figure 2. $a-c$, Significant activations in the ACC, insula, and SII. Areas shaded in blue were significantly more activated in response to identical pain stimuli when they were perceived to be uncontrollable rather than controllable $(U C-C)$. Areas shaded in green were significantly activated in both the uncontrollable and controllable conditions but were significantly more activated when the pain was perceived to be uncontrollable [(UC and $C)$ and $(U C-C)]$.

five subjects picked 5 , five subjects picked 4 , three subjects picked 3 , and three subjects picked 2 . This variability was found to be insignificant when included as a covariate in the fMRI analysis and was therefore not considered in subsequent analyses.

The mean pain rating was 7.19 (SD, 0.99) in the uncontrollable condition and 7.20 (SD, 1.00) in the controllable condition. This difference was not significant $\left(t_{(1,15)}=-0.086 ; p<0.9\right)$, suggesting that differences between these two conditions in the fMRI analysis were not caused by simple differences in the level of perceived pain.

\section{fMRI}

We compared activation in the two conditions by subtracting activation in the controllable condition from activation in the uncontrollable condition ( $\mathrm{UC}-\mathrm{C}$ ). Because the two conditions differed only in terms of the perception of controllability, the activations observed in this comparison were in no way related to any physical properties of the thermal stimulus or to any stimulus-driven affective response unrelated to the controllability manipulation. Previous imaging studies have consistently found activation in insular, secondary somatosensory, and anterior cingulate cortices in response to peripheral nociceptive input (Peyron et al., 2000). We found widespread bilateral activation in all of these regions in response to the controllability manipulation, as demonstrated in Figure $2 a-c$ (blue and green shading) and Table 1 . In addition to these areas, activation was observed in several regions that have been linked previously with pain, cognition, and affect processing, including the amygdala, hippocampus, thalamus, prefrontal cortex, and cerebellum (Table 1).

To examine the overlap of the neural responses to perceived controllability and peripheral stimulation, we isolated areas that were activated in both the controllable and uncontrollable pain conditions (UC and $\mathrm{C}$ ) and therefore responsive to the painful stimulus itself, independent of the controllability manipulation.
Table 1. Maximally activated voxels in clusters activated by controllability manipulation (UC - C)

\begin{tabular}{lll}
\hline Area & MNI coordinates $(x, y, z)$ & Zscore \\
\hline Controllability manipulation (UC - C) & & \\
Rostral ACC & $2,38,-2$ & 4.121 \\
& $-14,40,10$ & 3.432 \\
Insula & $40,-16,12$ & 5.345 \\
& $-44,-16,12$ & 3.645 \\
SII & $56,-12,12$ & 5.432 \\
& $-64,-16,16$ & 4.547 \\
Amygdala & $22,-6,-20$ & 3.403 \\
& $-24,-6,-18$ & 3.376 \\
Hippocampus/amygdala & $-18,-8,-22$ & 4.064 \\
Hippocampus & $-22,-16,-22$ & 3.876 \\
Thalamus & $-8,-12,4$ & 3.233 \\
Periaqueductal gray & $2,-38,-16$ & 4.793 \\
Prefrontal cortex (BA 10) & $26,52,0$ & 3.314 \\
Prefrontal cortex (BA 46) & $-42,36,12$ & 3.404 \\
Prefrontal cortex (BA 9) & $-28,34,42$ & 4.359 \\
SI/MI & $-36,-28,58$ & 4.745 \\
Cerebellum & $0,-52,-20$ & 4.158 \\
& $-10,-52,-50$ & 4.121 \\
& $-22,-56,-34$ & 4.281 \\
& $-10,-48,-30$ & 4.181 \\
Stimulus- controllability overlap [(UC and C) & and $(U C-C)]$ & 4.531 \\
Dorsal ACC (Fig. 3a) & $4,8,32$ & 4.411 \\
Insula/SII (Fig. 3b) & $-10,6,34$ & \\
Insula & $40,8,-6$ & 6.227 \\
SII & $-36,6,8$ & 3.770 \\
Medial frontal gyrus (BA 6) & $-54,8,-2$ & 4.715 \\
Cerebellum & $8,10,58$ & 4.165 \\
Con & $-32,-56,-28$ & 3.485 \\
& & 3.793 \\
\hline
\end{tabular}

'Controllability manipulation (UC $-C$ )' indicates areas activated only by the controllability manipulation and no by the stimulus itself. 'Stimulus - controllability overlap' $[(U C$ and $C)$ and $(U C-C)]$ indicates areas where processing of the nociceptive stimulus and controllability manipulation overlap. BA, Brodmann's area; MI, primary motor cortex; SI, primary somatosensory cortex.

We then isolated areas within these functionally defined regions of interest that were modulated by perceived controllability. The resulting map indicated that areas of insular, secondary somatosensory, and anterior cingulate cortices were significantly activated in response to the thermal stimulus in both conditions but were significantly more activated in the uncontrollable condition [(UC and C) and (UC - C)] (Figs. $2 a-c, 3 a, b$; Table 1). These results show that activation in neural areas frequently associated with pain is modulated by perceived controllability and that the belief that pain is controllable results in attenuated activation within these areas in response to nociceptive stimulation.

\section{Discussion}

The activations observed in the insular and secondary somatosensory cortices in this study are consistent with research in the domains of affect processing (Davidson and Irwin, 1999; Critchley et al., 2004) and pain processing (Craig, 2002), which converges to suggest that these areas play a role in interoception, maintaining a physical and emotional representation of the state of the body. Convergence of contextual and interoceptive information in the insula (indicating an influence of higher-level cognitive input on this somatic representation) was bilateral but predominant in anterior areas of the right insula (Fig. $2 b$, green shading, Fig. $3 b$ ). This pattern of activation is consistent with theoretical and anatomical work suggesting that although the insula receives somatic input bilaterally, a lateralized rerepresentation of the body is formed in the right anterior area (Craig, 
a

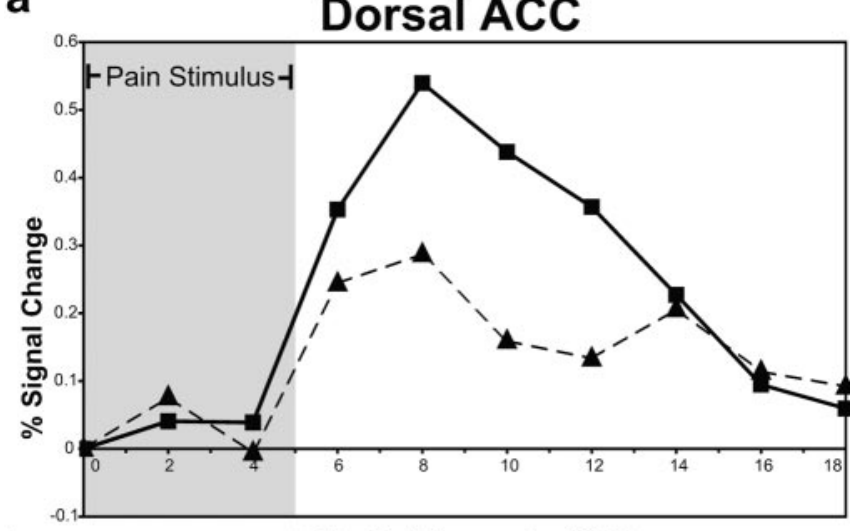

b

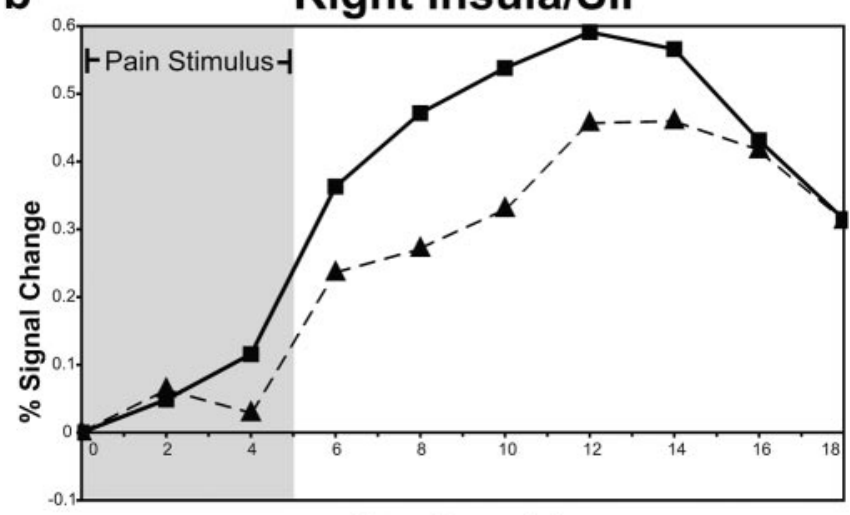

Time (Seconds)

Figure 3. $a, b$, Percentage of blood oxygenation level-dependent (BOLD) signal change over time in stimulus-controllability overlap clusters. Time-series plots for clusters in the dorsal ACC (a) and right insula-SII ( $b$ ) that were significantly activated by the stimulus (UC and $C$ ) and the controllability manipulation ( $U C-C$ ) (areas corresponding to the green shading in Fig. 2a-c) are shown. Percentage of signal change is measured from the beginning of the thermal stimulus and represents change from mean BOLD response across all time points in the experiment. The gray shading indicates the presence of the thermal stimulus.

2002), possibly as the foundation of the "emotional self" (Damassio, 1993). Thus, the finding that activation in this area is associated not only with sensory qualities of the nociceptive stimulus but also with perceived controllability suggests that this region may be critically involved in integrating interoceptive and contextual information necessary for higher-order somatic representations.

Rather than supporting the notion that the dorsocaudal area of the ACC activated in most pain imaging studies is a "nociceptive area" in the strictest sense, these data support an integrative role. This region (Fig. $2 a$, in green) was activated by the stimulus (independent of the controllability manipulation) but was significantly more activated when subjects believed the pain was uncontrollable. In light of previously hypothesized roles for this area in the affective and motivational aspect of pain (Rainville et al., 1997; Craig, 2003) and executive processes related to response (Devinsky et al., 1995; Carter et al., 1999), these data suggest that information about relevant contextual variables is integrated with sensory information in this area to facilitate contextappropriate affective and behavioral responses.

On the basis of both functional and cytoarchitectural considerations, it has been argued that the ACC should not be consid-

ered as a unitary structure but as a set of separate processing modules (Bush et al., 2000). These results are consistent with a functional subdivision of the ACC in pain processing. Whereas information about the peripheral stimulus and the degree of controllability appears to intersect in the dorsal region, the rostroventral portion of the ACC was only significantly activated by the controllability manipulation (Fig. 2a). This suggests that rather than processing information about the nociceptive stimulus, the rostroventral ACC may play a modulatory role based on contextual information such as perceived controllability. This is supported by the lack of consistent activation in this area in response to nociceptive stimulation in other pain imaging studies (Peyron et al., 2000) and by the involvement of this region in placebo analgesia (Petrovic et al., 2002) and cognitive modulation of pain (Bantick et al., 2002; Petrovic and Ingvar, 2002).

These findings not only suggest the powerful influence that cognitive factors such as perceived controllability have on the pain response but also have important implications for the generalizability of pain imaging studies. Nociceptive stimulation presented in the context of a typical pain imaging study represents uncontrollable pain. Subjects are commonly "trapped" within a PET or MRI scanner, given no behavioral option for controlling the pain they feel, and instructed not to move. The results presented here suggest that the patterns of activation observed in the ACC, insula, and SII might be attributable in part to cognitive processes specific to uncontrollable pain. As an example, it is possible, given the hypothesized involvement of the ACC in response selection (Devinsky et al., 1995), that the activation observed in this region may be attributable in part to response inhibition, as the subject suppresses the impulse to withdraw from painful stimulation in the imaging environment. On the basis of our findings, we suggest that some pain imaging studies may overestimate the extent to which activation in these areas is caused by stimulus-driven processes and common across all cognitive contexts.

These results suggest two areas for further study. First, it is important to understand the processes that may be responsible for the observed effects and how these processes relate to pain perception. The lack of a difference between pain ratings in the two conditions suggests that the differences in neural activity are not attributable to simple differences in pain perception. Although it is possible that the analysis of self-reported pain perception was simply underpowered, the lack of a trend in either direction suggests that this interpretation is unlikely. What is more likely is that the observed differences in neural activity reflect cognitive and affective processes that are associated with controllability but may be either unrelated to pain perception or inconsistent between subjects. In all likelihood, there are individual differences in the way that subjects respond to perceived uncontrollability. Given the strong modulatory effect of attention on the neural response to pain (Bantick et al., 2002), the observed differences between the conditions could be attributable in part to differences between the conditions in the degree to which subjects attended to the pain stimulus. Although the present study did not formally assess the strategy used by subjects to cope with the uncontrollable pain, it is plausible that some subjects attempted to distract themselves from the pain, whereas others attended more strongly to the nociceptive stimulation. These strategies might both be associated with increased neural activation but could have very different effects on pain perception.

A second area for future study is examination of the potential clinical implications of perceived controllability over pain. Chronic pain disorders are frequently comorbid with affective 
disorders such as depression. Lack of perceived control over negative events (including pain) can strongly influence outcomes in both types of disorders (Seligman, 1975; Jensen and Karoly, 1991). The present study found that information about perceived controllability has a profound influence on the processing of pain in areas such as the insular and anterior cingulate cortices that have been linked previously with both adaptive and maladaptive affective processing (Davidson and Irwin, 1999; Davidson et al., 2002). Continued study of how perceived controllability modulates the neural response to pain may therefore help to illuminate the relationship between these puzzling and debilitating disorders.

\section{References}

Bantick SJ, Wise RG, Ploghaus A, Clare S, Smith SM, Tracey I (2002) Imaging how attention modulates pain in humans using functional MRI. Brain 125:310-319.

Beckmann C, Jenkinson M, Smith S (2003) General multilevel linear modeling for group analysis in FMRI. NeuroImage 20:1052-1063.

Bush G, Luu P, Posner MI (2000) Cognitive and emotional influences in anterior cingulate cortex. Trends Cogn Sci 4:215-222.

Carter CS, Botvinick MM, Cohen JD (1999) The contribution of the anterior cingulate cortex to executive processes in cognition. Rev Neurosci 10:49-57.

Craig AD (2002) How do you feel? Interoception: the sense of the physiological condition of the body. Nat Rev Neurosci 3:655-666.

Craig AD (2003) A new view of pain as a homeostatic emotion. Trends Neurosci 26:303-307.

Critchley HD, Wiens S, Rotshtein P, Ohman A, Dolan RJ (2004) Neural systems supporting interoceptive awareness. Nat Neurosci 7:189-195.

Damassio AR (1993) Descartes' error: emotion, reason and the human brain. New York: Putnam.

Davidson RJ, Irwin W (1999) The functional neuroanatomy of emotion and affective style. Trends Cogn Sci 3:11-21.

Davidson RJ, Pizzagalli D, Nitschke JB, Putnam K (2002) Depression: perspectives from affective neuroscience. Annu Rev Psychol 53:545-574.

Devinsky O, Morrell MJ, Vogt BA (1995) Contributions of anterior cingulate cortex to behaviour. Brain 118:279-306.

Forman SD, Cohen JD, Fitzgerald M, Eddy WF, Mintun MA, Noll DC (1995) Improved assessment of significant activation in functional magnetic res- onance imaging (fMRI): use of a cluster-size threshold. Magn Reson Med 33:636-647.

Friston KJ, Worsley RS, Frakowiak J, Mazziotta JC, Evans AC (1994) Assessing the significance of focal activations using their spatial extent. Hum Brain Mapp 1:214-220.

Jenkinson M, Bannister P, Brady M, Smith S (2002) Improved optimization for the robust and accurate linear registration and motion correction of brain images. NeuroImage 17:825-841.

Jensen MP, Karoly PJ (1991) Control beliefs, coping efforts, and adjustment to chronic pain. J Consult Clin Psychol 59:431-438.

Jensen MP, Turner JA, Romano JM (2001) Changes in beliefs, catastrophizing, and coping are associated with improvement in multidisciplinary pain treatment. J Consult Clin Psychol 69:655-662.

Maier SF, Watkins LR (1998) Stressor controllability, anxiety and serotonin. Cognit Ther Res 22:595-613.

Melzack R, Casey KL (1968) Sensory, motivational, and central control determinants of pain: a new conceptual model. In: The skin senses (Kenshalo DR, ed), pp 423-439. Springfield, IL: Thomas.

Mineka S, Henderson RW (1985) Controllability and predictability in acquired motivation. Annu Rev Psychol 36:495-529.

Petrovic P, Ingvar M (2002) Imaging cognitive modulation of pain processing. Pain 95:1-5.

Petrovic P, Kalso E, Petersson KM, Ingvar M (2002) Placebo and opioid analgesia-imaging a shared neuronal network. Science 295:1737-1740.

Peyron R, Laurent B, Garcia-Larrea L (2000) Functional imaging of brain responses to pain. Neurophysiol Clin 30:263-288.

Ploghaus A, Tracey I, Gati JS, Clare S, Menon RS, Matthews PM, Rawlins JN (1999) Dissociating pain from its anticipation in the human brain. Science 284:1979-1981.

Ploghaus A, Becerra L, Borras C, Borsook D (2003) Neural circuitry underlying pain modulation: expectation, hypnosis, placebo. Trends Cogn Sci 7:197-200

Rainville P (2002) Brain mechanisms of pain affect and pain modulation. Curr Opin Neurobiol 12:195-204.

Rainville P, Duncan GH, Price DD, Carrier B, Bushnell MC (1997) Pain affect encoded in human anterior cingulate but not somatosensory cortex. Science 277:968-971.

Seligman MEP (1975) Helplessness: on depression, development, and death. San Francisco: Freeman.

Worsley KJ, Evans AC, Marrett S, Neelin PA (1992) Three-dimensional statistical analysis for CBF activation studies in human brain. J Cereb Blood Flow Metab 12:900-918. 